\title{
General Method for Incorporating CALPHAD Free Energies of Mixing into Phase Field Models: Application to the $\alpha$-Zirconium $/ \delta$-Hydride System
}

\author{
A. M. Jokisaari, K. Thornton \\ Department of Materials Science and Engineering, College of Engineering, University of Michigan, Ann Arbor, MI \\ 48109, USA
}

\begin{abstract}
This paper presents a general method of incorporating CALPHAD-based free energies into a phase field model. While CALPHAD-based free energy descriptions provide realistic energetics of alloys, their formulations may pose numerical difficulties for phase field simulations. Specifically, the free energies of mixing for different phases may not necessarily be defined over the same concentration ranges, and their derivatives may exhibit highly nonlinear behavior. The method presented approximates the free energies of mixing using piecewise functions to eliminate the aforementioned characteristics while largely retaining the free energy values and the first and second derivative information, which affect the thermodynamic and kinetic behavior of the system. The method is verified by planar interface simulations of the $\alpha$-zirconium $/ \delta$-hydride system. The phase fractions and compositions obtained from the phase field simulations are compared to the values calculated from the common tangent construction on the original free energies. The results indicate a high degree of accuracy.
\end{abstract}

CALPHAD; phase field model; finite element; zirconium; zirconium hydride

\section{Introduction}

Quantitative microstructural modeling of phase transformations such as solidification 
and second-phase precipitation is of major technological and scientific importance for materials development and design. One means of modeling microstructural evolution is the phase field approach, which has been successfully employed to simulate phase transformations such as spinodal decomposition [1--4], coarsening [5, 6], solidification [7--9], and thin film growth [3, 10--13]. Comprehensive descriptions and reviews of phase field modeling are found in Refs. [14--18]. In a phase field model, a microstructure is described by one or more continuous conserved or nonconserved field variables, termed order parameters. An order parameter is generally denoted as $\psi(\mathbf{r}, t)$ and indicates the phase at $\mathbf{r}$, where $\mathbf{r}$ is position and $t$ is time. Each phase is designated by a bulk value (e.g., $\psi=1$ for the $\alpha$ phase and $\psi=0$ for the $\beta$ phase), and the value of $\psi$ changes smoothly between the phases. The position of the interface between the phases is described by an intermediate value (e.g., $\psi=0.5$ ). Thus, the phase field methodology eliminates the need to track the positions of the interfaces explicitly. The free energy of the system can be described as a functional of the order parameters, and the evolution of the system is driven by the reduction of the free energy $[4,19]$.

The CALPHAD method is a semi-empirical approach for formulating free energies of mixing using known thermodynamic data and equilibrium phase diagrams [20, 21]. The incorporation of realistic CALPHAD-based free energies into phase field models has significantly increased prediction capabilities of phase field modeling [14--16]. To date, this approach has been applied to steels [22--25], superalloys [26--33], and aluminum alloys [34--36], among others, with studies examining both solidification [22, 26, 32, 34, 36--42] and solid-state transformations [23--31, 33, 35, 43--45].

While CALPHAD-based free energy descriptions may provide realistic thermodynamic information, the presence of natural logarithm terms in their formulation poses numerical challenges for simulation. For example, Ref. [46] indicated the difficulty in performing phase field simulations at low vacancy concentrations for a TiAlN-vacancy system described by CALPHAD-based free energies. In addition, the free energies of mixing may 
only be defined over a finite concentration range of solute and their derivatives may exhibit undesirable asymptotic behavior. Furthermore, free energies of mixing for different phases may not be defined over the same concentration ranges (e.g., $G^{\alpha}$ may be defined over an atomic fraction range of $(0,0.5)$, while $G^{\beta}$ may be defined over $\left.(0,1)\right)$. These attributes may be problematic for numerical simulations because solvers may attempt to compute a free energy for a concentration value outside the range for which it is defined. In many phase field models (e.g., the Wheeler-Boettinger-McFadden (WBM) [47], Kim-Kim-Suzuki (KKS) [48], or Welland-Wolf-Guyer (WWG) [49] models), free energy curves must be defined over a large composition range, even at compositions far from equilibrium. However, the computed mobility may be negative when it is calculated from the experimentally obtained diffusivities and the second derivative of the free energy (e.g., in the spinodal region). To avoid such a situation, the CALPHAD free energy must be modified.

Previously proposed methods of incorporating CALPHAD-based free energies into phase field models include direct coupling to a thermodynamic database $[26,50,51]$ or polynomial approximations of the free energies [35, 52--55]. However, direct coupling may incur substantial computational expense by querying external software at each mesh point for every simulation step, and it may necessitate the use of commercial software, which may require costly licensing fees. Approximating the free energy as a polynomial expression alleviates the aforementioned computational cost, but a simple approximation may cause a loss of thermodynamic information. For example, Ref. [35] found that precipitate growth kinetics and the driving force for nucleation are sensitive to the parameterization of the free energy of the matrix and precipitate phases, respectively, in an Al-Cu system.

The $\alpha$-zirconium $/ \delta$-hydride system is of technological importance, particularly for the fuel assemblies of nuclear power reactors. A CALPHAD-based description of the molar free energies of mixing for this system exists in the literature [56]. However, the free energies exhibit several characteristics that are problematic for phase field modeling, including the presence of natural logarithms arising from the entropic contribution to the 
free energy. In addition, the solubility limit of hydrogen in $\alpha$-zirconium is low. Therefore, at low supersaturations a numerical solver may attempt to compute a free energy for a hydrogen atomic fraction less than zero, resulting in undefined behavior due to the natural logarithm terms. Furthermore, the $\alpha$-phase free energy of mixing is defined over a smaller composition range than that of the $\delta$-phase, so that energy evaluations may occur outside the composition range of the $\alpha$-phase but still within the composition range of the $\delta$-phase.

In this paper, a simple and efficient method of approximating CALPHAD-based free energies for phase field models is proposed. The approximation method was formulated to retain the original free energy in the composition ranges that are present in the evolving system while alleviating numerical challenges. The approach is incorporated into a phase field model for the $\alpha$-zirconium $/ \delta$-hydride system, which was chosen as a test system because it exhibits the characteristics described previously. Planar interface simulations were performed to verify the model. The equilibrium phase fractions and compositions from the simulations were compared to values obtained from the original CALPHAD free energies via a common tangent construction. In addition, an example of $\delta$-hydride precipitate growth is discussed to demonstrate the flexibility of the method.

\section{Methods}

To incorporate a CALPHAD-based free energy functional in a phase field model, an implementation must effectively handle any instances where the free energies of mixing for the phases become undefined or discontinuous. These instances may be treated either by numerical exceptions or by modifying the functional such that it yields numerically acceptable behavior while retaining essential thermochemical information. In this paper, we take the latter approach. Below, we first describe a general phase field model to provide the form of the free energy required. We then provide the generic framework for approximating the free energies of mixing. 


\subsection{General coupled conserved-nonconserved phase field model}

In the phase field approach, the state of a two-phase system may be described using two field variables. A conserved field variable, $X$, describes the atomic fraction of solute, and a nonconserved structural field variable, $\eta$, tracks the structural transformation between the two phases. The free energy, $G$, is expressed as

$$
G=\int_{V}\left(g_{\text {chem }}(X, \eta, T)+\frac{\kappa_{X}}{2}|\nabla X|^{2}+\frac{\kappa_{\eta}}{2}|\nabla \eta|^{2}\right) d V,
$$

where $\kappa_{\eta}$ and $\kappa_{X}$ are the gradient energy coefficients for the structural order parameter and atomic fraction, respectively, $g_{\text {chem }}$ is the bulk chemical free energy density, $T$ is the absolute temperature, and $V$ is the system volume. Additional energy contributions may be incorporated into the free energy as well. In phase field model formulations suited for incorporating CALPHAD-based free energies (including the WBM, KKS and WWG models), the expression for $g_{c h e m}$ is a combination of two single phase energies,

$$
g_{\text {chem }}=\frac{1}{\Omega_{0}}\left[(1-h(\eta)) G_{m i x}^{0, \alpha}+h(\eta) G_{m i x}^{0, \beta}+w k(\eta)\right],
$$

where $G_{\text {mix }}^{0, \alpha}$ is the molar free energy of mixing for the solute in the $\alpha$-phase and $G_{\text {mix }}^{0, \beta}$ is the molar free energy of mixing for the $\beta$-phase. Note that $G_{m i x}^{0, \alpha}$ and $G_{m i x}^{0, \beta}$ are dependent on composition and temperature, but their exact dependence varies depending on the phase field model employed (e.g., $G_{m i x}^{0, \alpha}(X, T)$ for the WBM model and $G_{m i x}^{0, \alpha}\left(X^{\alpha}, T\right)$ for the KKS model, where $X^{\alpha}$ is the composition in the $\alpha$ phase. In addition, $\Omega_{0}$ is the molar volume, $h(\eta)=\eta^{2}(3-2 \eta)$ is the interpolation function that increases monotonically from $h(0)=0$ to $h(1)=1, k(\eta)=\eta^{2}(\eta-1)^{2}$ is the double well function, and $w$ controls the height of the barrier in the double well function, though other terms may contribute to the total energy barrier.

The microstructural evolution of the system is governed by coupled conserved-nonconserved 
dynamics [47]. The Cahn-Hilliard equation [4] governs the evolution of the atomic fraction of solute. To reduce computational resource requirements, the Cahn-Hilliard equation may be split into two second-order equations $[57,58]$. The time evolution of $X$ is given by

$$
\frac{1}{\Omega_{0}} \frac{\partial X(\mathbf{r}, t)}{\partial t}=\nabla \cdot M \nabla \frac{\delta G}{\delta X^{\prime}}
$$

where $M$ is the mobility, and

$$
\frac{\delta G}{\delta X}=\frac{\partial g_{\text {chem }}}{\partial X}-\kappa_{X} \nabla^{2} X
$$

When $\kappa_{X}=0$, the form of the phase field model in Ref. [47] is recovered; note that a non-zero value of $\kappa_{X}$ may be required to control the maximum gradient of $X$. In addition, the Allen-Cahn equation [19] governs the evolution of the structural order parameter as

$$
\frac{\partial \eta(\mathbf{r}, t)}{\partial t}=-L \frac{\delta G}{\delta \eta}=-L\left(\frac{\partial g_{\text {chem }}}{\partial \eta}-\kappa_{\eta} \nabla^{2} \eta\right)
$$

where $L$ is the kinetic coefficient.

\subsection{Generic CALPHAD free energy approximation}

In the CALPHAD method, a molar free energy of mixing for a single phase is expressed in the form

$$
G_{\text {mix }}^{0, i}(X, T)=G_{r e f}^{0, i}(X, T)+G_{i d e a l}^{0, i}(X, T)+G_{\text {excess }}^{0, i}(X, T),
$$

where $G_{r e f}^{0, i}$ is the reference free energy of mixing of the "end members" [21], $G_{\text {ideal }}^{0, i}$ is the free energy of mixing for an ideal solution, and $G_{\text {excess }}^{0, i}$ is the free energy of mixing that deviates from the ideal solution model [20]. The end members may be elements or stoichiometric compounds, as in the case of free energies formulated according to a sublattice model [21]. The sublattice model is a common technique for developing free 
energies of mixing for substitutional and interstitial solutions as well as compounds [21].

The equation for $G_{\text {mix }}^{0, i}$ presents several difficulties for numerical discretization. Depending on the exact energy formulation, the presence of the natural logarithm terms in $G_{i d e a l}^{0, i}$ may result in $G_{\text {mix }}^{0, i}$ not being defined over the entire atomic fraction range of $(0,1)$ but only a subset (e.g., $(0,0.5))$. For example, the sublattice model may encounter this issue. If $G_{m i x}^{0, \alpha}$ and $G_{m i x}^{0, \beta}$ are defined over different atomic fraction ranges, then the phase field formulation for the chemical free energy density (Eq. 2) may require calculation of energies outside the composition range of one $G_{\text {mix }}^{0, i}$ but within the composition range of the other. In addition, both $\partial G_{m i x}^{0, \alpha} / \partial X$ and $\partial G_{m i x}^{0, \beta} / \partial X$ exhibit highly nonlinear behavior near their composition bounds due to the natural logarithm terms in $G_{i \text { deal }}^{0, \alpha}$ and $G_{i d e a l}^{0, \beta}$. Finally, the numerical solution method may attempt to calculate energies for $X<0$. Therefore, either numerical exception handling or free energy reformulation to avoid these undefined values is necessary.

To eliminate the instances in which Eq. 2 becomes undefined, we approximate $G_{m i x}^{0, i}(X, T)$ as a piecewise function $\tilde{G}_{\text {mix }}^{0, i}(X, T)$, where $i=\alpha$ or $\beta$. This approximation is given by Eq. 7 and is schematically illustrated in Fig. 1. Each piecewise function $\tilde{G}_{m i x}^{0, i}(X, T)$ is composed of three subfunctions that are defined on subdomains of $X$. The low and high subdomain boundary compositions ( $X_{\text {low }}$ and $X_{h i g h}$, respectively) are chosen within the composition domain over which each $G_{\text {mix }}^{0, i}(X, T)$ is defined. These subdomain boundary compositions (collectively referred to as $X_{\text {bound }}$ ) must be selected to be sufficiently outside the composition range expected to be encountered in the physical system. The free energy for $X<X_{\text {low }}$ is approximated by the second-order Taylor expansion of $G_{m i x}^{0, i}(X, T)$ around $X_{\text {low }}$, denoted as $G_{\text {Taylor }}^{\text {low }}$. Similarly, the second-order Taylor expansion of $G_{\text {mix }}^{0, i}(X, T)$ around $X_{\text {high }}$ is used to approximate $G_{\text {mix }}^{0, i}(X, T)$ for $X>X_{\text {high }}$, denoted as $G_{\text {Taylor }}^{\text {high }}$. The resulting function retains the derivatives of the free energy within the $X_{\text {bound }}$ values and creates a new energy that is second-order differentiable for all composition and temperature ranges, as long as the original CALPHAD energies are second-order differentiable. 


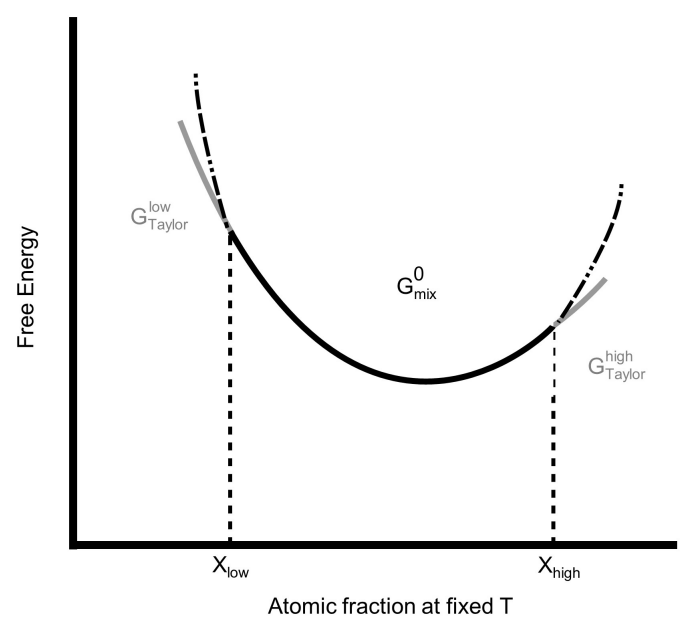

Figure 1: Schematic illustration of the approximation method. The original CALPHAD-based free energy is labeled $G_{m i x}^{0, i}$ (black curve). At and below the subdomain boundary composition $X_{\text {low }}, G_{\text {Taylor }}^{\text {low }}$ is substituted for $G_{m i x}^{0, i}$, and at and above the composition $X_{\text {max }}, G_{\text {Taylor }}^{\text {high }}$ is substituted (two gray curves). The functions $G_{\text {Taylor }}^{\text {low }}$ and $G_{\text {Taylor }}^{\text {high }}$ are the second-order Taylor expansions of $G_{\text {mix }}^{0, i}(X, T)$ at $X_{l o w}$ and $X_{h i g h}$, respectively. The approximated function $\tilde{G}_{\text {mix }}^{0, i}$ is given by the combination of the solid gray and black curves. The dashed lines indicate where $G_{\text {mix }}^{0, i}$ is discarded.

The resulting expression for $\tilde{G}_{\text {mix }}^{0, i}(X, T)$ is given by

$$
\tilde{G}_{\text {mix }}^{0, i}(X, T)= \begin{cases}\left.G_{\text {mix }}^{0, i}(X, T)\right|_{X=X_{\text {low }}} & \\ +\left.\frac{\partial G_{\text {mix }}^{0, i}(X, T)}{\partial X}\right|_{X=X_{\text {low }}}\left(X-X_{\text {low }}\right) & \\ +\left.\frac{1}{2} \frac{\partial^{2} G_{\text {mix }}^{0, i}(X, T)}{\partial X^{2}}\right|_{X=X_{\text {low }}}\left(X-X_{\text {low }}\right)^{2} & : X<X_{\text {low }} \\ G_{\text {mix }}^{0, i}(X, T) & : X_{\text {low }} \leq X \leq X_{\text {high }} \leq \\ \left.G_{\text {mix }}^{0, i}(X, T)\right|_{X=X_{\text {high }}} & \\ +\left.\frac{\partial G_{\text {mix }}^{0, i}(X, T)}{\partial X}\right|_{X=X_{\text {high }}}\left(X-X_{\text {high }}\right) & \\ +\left.\frac{1}{2} \frac{\partial^{2} G_{\text {mix }}^{0, i}(X, T)}{\partial X^{2}}\right|_{X=X_{\text {high }}}\left(X-X_{\text {high }}\right)^{2} & : X>X_{\text {high }} .\end{cases}
$$




\subsection{The $\alpha$-zirconium/ $\delta$-hydride model}

\subsubsection{Phase field model formulation}

The CALPHAD free energy approximation method is demonstrated for the $\alpha$-zirconium $/ \delta$ hydride system. In this model, $X$ is the atomic fraction of hydrogen and $\eta$ tracks the structural transformation between the $\alpha$-zirconium and $\delta$-hydride phases, where $\eta=0$ for the $\alpha$ phase and $\eta=1$ for the $\delta$ phase. Zirconium hydride precipitation in zirconium induces elastic strains [59], but chemical and elastic energy contributions are typically incorporated separately in a phase field model [18]. Therefore, we neglect the elastic strain energy contribution to focus on the verification of the chemical free energy calculation. The effect of elastic energy will be considered in a future publication. The chemical free energy is written as

$$
g_{\text {chem }}=\frac{1}{\Omega_{0}}\left[(1-h(\eta)) G_{\text {mix }}^{0, \alpha}(X, T)+h(\eta) G_{\text {mix }}^{0, \delta}(X, T)+w k(\eta)\right],
$$

where $G_{\text {mix }}^{0, \alpha}(X, T)$ is the molar free energy of mixing of $\alpha$-zirconium with hydrogen, $G_{m i x}^{0, \delta}(X, T)$ is the molar free energy for $\delta$-hydride, and $\Omega_{0}=1.4 \times 10^{-5} \mathrm{~m}^{3} / \mathrm{mol}$ is the molar volume of $\alpha$-zirconium. The microstructural evolution of the system is governed by Eqs. 3 and 5.

While quantitative values of the $\alpha / \delta$ interfacial energies are not available in the literature, experimental evidence indicates that nanoscale hydrides are semi-coherent with the matrix [59] while larger hydrides are incoherent [60]. Therefore, the interfacial energy was estimated according to

$$
\gamma=\gamma_{c h}+\gamma_{s t}
$$

where $\gamma_{c h}$ is the chemical energy of the interface and $\gamma_{s t}$ is the energy due to the presence of dislocations reducing the misfit strain between the $\alpha$ and $\delta$ phases. We chose $\gamma_{c h}=$ 
$35 \mathrm{~mJ} / \mathrm{m}^{2}$, which was calculated in Ref. [61] for other coherent zirconium hydride phases. The dislocation contribution to the interfacial energy was obtained in Ref. [62] as $\gamma_{s t} \approx$ $\frac{1}{2} S b \epsilon^{0}$, where $S$ is the shear modulus of zirconium, $b$ is the Burgers vector of a dislocation, roughly equal to the lattice constant of $\delta$-hydride, and $\epsilon^{0}$ is the average $\alpha / \delta$ misfit strain [63]. However, experimental evidence indicates that the misfit strain is not fully relieved by interfacial dislocations for nanoscale hydrides [59]. To model this case, we introduce $\lambda$, which is the fraction of the misfit strain relieved by interfacial dislocations and takes a value between zero and one, such that $\gamma_{s t} \approx \frac{1}{2} S b \lambda \epsilon^{0}$. A smaller $\lambda$ value indicates a larger dislocation spacing. To represent an intermediate state with respect to the fully coherent interface and the fully relaxed interface, $\lambda=0.5$ was chosen such that $\gamma_{s t} \approx 280 \mathrm{~mJ} / \mathrm{m}^{2}$ and $\gamma=305 \mathrm{~mJ} / \mathrm{m}^{2}$. Due to the WBM model formulation of the free energy density in Eq. 8 , an inherent interfacial energy contribution exists even when $w=0$, limiting the ability to control the diffuse interface width for a given interfacial energy. For $w=0, \kappa_{X}=0$, and this interface energy, $\kappa_{n}=4 \times 10^{-10} \mathrm{~J} / \mathrm{m}$ was found numerically, resulting in a diffuse interface width of $1 \mathrm{~nm}$ when measured between $0.1<\eta<0.9$.

The mobility of hydrogen in the system must be formulated such that the diffusivity of hydrogen in the $\alpha$ and $\delta$ phases is reproduced in the phase field model. In addition, the mobility must be positive [64]. For a single phase, the flux $\mathbf{J}$ of hydrogen calculated according to Fick's first law must match the flux in the phase field model,

$$
\mathbf{J}=-D \nabla\left(\frac{X}{\Omega_{0}}\right)=-M \nabla\left(\frac{1}{\Omega_{0}} \frac{\partial G_{m i x}^{0, i}}{\partial X}\right)
$$

where $D$ is the diffusivity. For the $\alpha$-zirconium $/ \delta$-hydride phase field model, the mobility in the $\alpha$ phase is given as

$$
M^{\alpha}(X, T)=\frac{D^{\alpha}(T)}{\partial^{2} \tilde{G}_{m i x}^{0, \alpha} / \partial X^{2}}
$$


and the mobility in the $\delta$ phase is given as

$$
M^{\delta}(X, T)=\frac{D^{\delta}(T)}{\partial^{2} \tilde{G}_{m i x}^{0, \delta} / \partial X^{2}}
$$

such that the total mobility in Eq. 3 is

$$
M(X, \eta, T)=(1-h(\eta)) M^{\alpha}(X, T)+h(\eta) M^{\delta}(X, T),
$$

where $D^{\alpha}(T)$ is the diffusivity of hydrogen in $\alpha$-zirconium [65] and $D^{\delta}(T)$ is the diffusivity of hydrogen in $\delta$-hydride [66].

\subsection{2. $C A L P H A D$ free energy approximations}

Both the $\alpha$-zirconium phase (solid solution with interstitial hydrogen) and the $\delta$-hydride phase can be modeled as two-sublattice systems, in which zirconium fills the first sublattice and hydrogen and vacancies fill the second sublattice $[67,68]$. The free energies of mixing for the $\alpha$-zirconium $/ \delta$-hydride system were parameterized by Dupin et al. [56] using the CALPHAD method. The molar free energy of mixing for hydrogen in $\alpha$-zirconium is given as

$$
\begin{aligned}
G_{m i x}^{0, \alpha}(X, T)= & {\left[(1-2 X) G_{Z r}^{0, h c p}(T)+X G_{Z r H}^{0, h c p}(T)\right] } \\
& +R T\left[X \ln \left(\frac{X}{1-X}\right)+(1-2 X) \ln \left(\frac{1-2 X}{1-X}\right)\right]
\end{aligned}
$$

and that for $\delta$-hydride is given as 


$$
\begin{aligned}
G_{\text {mix }}^{0, \delta}(X, T)= & \frac{1}{2}\left[(2-3 X) G_{Z r}^{0, f c c}(T)+X G_{Z r H_{2}}^{0, f c c}(T)\right] \\
& +R T\left[X \ln \left(\frac{X}{2(1-X)}\right)+(2-3 X) \ln \left(\frac{2-3 X}{2(1-X)}\right)\right] \\
& +\left(\frac{3 X^{2}-2 X}{4(X-1)^{2}}\right)\left[(X-1) L_{f c c}^{0}(T)+(1-2 X) L_{f c c}^{1}(T)\right]
\end{aligned}
$$

where $R$ is the gas constant [56]. The parameterizations for $G_{Z r H}^{0, h c p}(T), G_{Z r H_{2}}^{0, f c c}(T), L_{f c c}^{0}(T)$, and $L_{f c c}^{1}(T)$ are supplied by Ref. [56], and the parameterizations for $G_{Z r}^{0, h c p}(T)$ and $G_{Z r}^{0, f c c}(T)$ are from Ref. [69]. We parameterized $G_{H_{2}}^{0, g a s}(T)$, which is needed to calculate $G_{\mathrm{ZrH}}^{0, h c p}(T)$ and $G_{\mathrm{ZrH}_{2}}^{0, f c c}(T)$, using the data in Ref. [70]. All of the parameterizations are reproduced in the Appendix.

The free energy $G_{\text {mix }}^{0, \alpha}$ is defined over the composition range of $X=(0,0.5)$, and $G_{\text {mix }}^{0, \delta}$ is defined over $X=\left(0, \frac{2}{3}\right)$, while the equilibrium atomic fraction of hydrogen in the $\delta$-hydride phase is approximately $X=0.6$. The phase field formulation for the chemical free energy density necessitates calculation of energies outside the composition range of $G_{m i x}^{0, \alpha}$ when the system is in the $\delta$-hydride phase. Equations 14 and 15 are approximated using Eq. 7 to obtain $\tilde{G}_{\text {mix }}^{0, \alpha}(X, T)$ and $\tilde{G}_{\text {mix }}^{0, \delta}(X, T)$, which are substituted for $G_{\text {mix }}^{0, \alpha}(X, T)$ and $G_{m i x}^{0, \delta}(X, T)$ in Eq. 8. Following the method in Section 2.2, four $X_{\text {bound }}$ compositions were chosen as $X_{\text {low }}^{\alpha}=0.001, X_{\text {high }}^{\alpha}=0.499, X_{\text {low }}^{\delta}=0.5$, and $X_{\text {high }}^{\delta}=0.665$. Figure 2 shows the surface of the chemical free energy density (Eq. 8) that results from using the approximated $\tilde{G}_{\text {mix }}^{0, \alpha}$ and $\tilde{G}_{m i x}^{0, \delta}$; the surface is continuous and differentiable everywhere.

The mobility must be a positive quantity. Given that diffusion coefficients are measured in a single phase and are thus always positive, the phase field formulation requires a positive second derivative of the free energy (thermodynamic factor) to obtain a positive, non-divergent hydrogen mobility (Eq. 13). The value for $X_{\text {low }}^{\delta}$ was chosen because the sign of the second derivative of $G_{\text {mix }}^{\delta, 0}(X, T)$ changes at $X \approx 0.48$. For $X \geq 0.5$, the second 


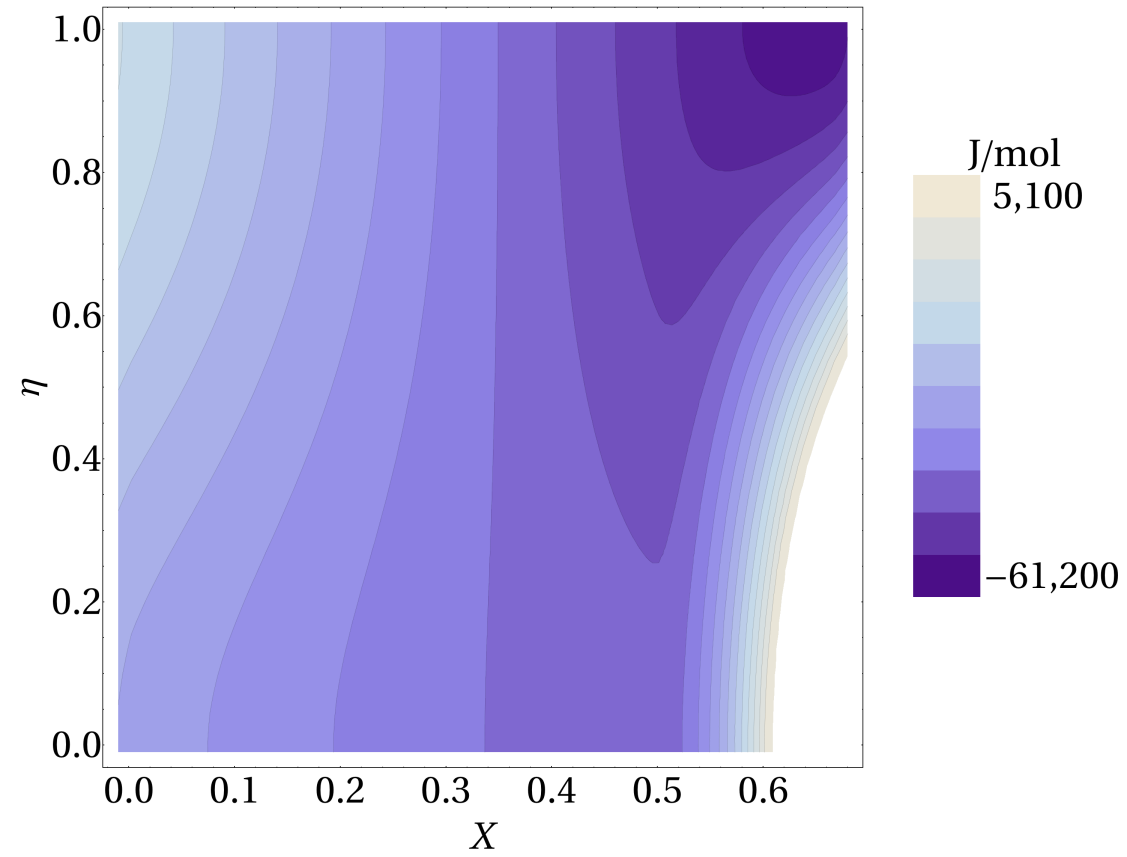

Figure 2: A contour plot of the free energy surface $f_{\text {chem }}(X, \eta, T)$ at $600 \mathrm{~K}$ formed by applying the approximation method to $G_{\text {mix }}^{0, \alpha}$ and $G_{\text {mix }}^{0, \delta}$.

derivative of $G_{m i x}^{\delta, 0}(X, T)$ is positive; by choosing $X_{\text {low }}^{\delta}=0.5, \tilde{G}_{m i x}^{\delta, 0}(X, T)$ exhibits a positive second derivative for all $X$ and all $T$ within $485 \mathrm{~K}$ to $750 \mathrm{~K}$.

\subsubsection{Nondimensionalization of the equations}

The governing equations are nondimensionalized in this work, which improves numerical behavior by ensuring all quantities are within approximately an order of unity. The nondimensionalization method adopted here follows that presented in Ref. [71]. Characteristic scale values for the system's length, energy, and time are defined, and the equations are nondimensionalized using these values. The characteristic length scale was set to $l=1 \times 10^{-9} \mathrm{~m}$ because we are interested in nanoscale phenomena. The characteristic volumetric energy scale was the maximum driving force for phase transformation at $593 \mathrm{~K},|\Delta f|=4.009 \times 10^{8} \mathrm{~J} / \mathrm{m}^{3}$. In addition, while no experimental information exists for the Allen-Cahn kinetic coefficient, $L=1 \times 10^{-5} \mathrm{~m}^{3} \mathrm{~J}^{-1} \mathrm{~s}^{-1}$ was chosen to maintain a diffusion-controlled transformation [35]. The relationships between the dimensional and 
dimensionless quantities are presented in Table 1, in which the dimensionless quantities are denoted with asterisks.

Table 1: Nondimensionalization expressions for quantities within the $\alpha$-zirconium $/ \delta$-hydride phase field model.

\begin{tabular}{|c|c|}
\hline Quantity & Nondimensionalization \\
\hline \hline Time & $t^{*}=L|\Delta f| t$ \\
\hline Length & $r^{*}=r / l$ \\
\hline Hydrogen phase field mobility & $M^{*}=M \Omega_{0} /\left(L l^{2}\right)$ \\
\hline Allen-Cahn kinetic coefficient & $L^{*}=L / L \equiv 1$ \\
\hline Hydrogen atomic fraction gradient coefficient & $\kappa_{X}^{*}=\kappa_{X} /\left(|\Delta f| l^{2}\right)$ \\
\hline Structural order parameter gradient coefficient & $\kappa_{\eta}^{*}=\kappa_{\eta} /\left(|\Delta f| l^{2}\right)$ \\
\hline Chemical energy & $f_{\text {chem }}^{*}=f_{\text {chem }} /|\Delta f|$ \\
\hline
\end{tabular}

\subsubsection{Simulation conditions}

To verify the approximation method, several planar interface simulations were run to equilibrium, i.e., uniform chemical potential, and the phase fractions and compositions were compared to the values obtained from the common tangent construction of $G_{\text {mix }}^{0, \alpha}(X, T)$ and $G_{\text {mix }}^{0, \delta}(X, T)$. A planar interface was chosen to eliminate the GibbsThomson effect, which shifts equilibrium phase compositions due to the presence of a curved interface. No-flux boundary conditions were applied for $X$ and $\eta$, and isothermal conditions with $T=485,550,600,650$, and $750 \mathrm{~K}$ were chosen. The lowest temperature, 485 $\mathrm{K}$, was chosen because $X_{\text {low }}^{\alpha}$ is approximately equal to the composition of the $\alpha$-zirconium phase boundary at that temperature and thus represents the lower limit for the applicability of the approximation. The highest temperature, $750 \mathrm{~K}$, is close to the $\beta$-zirconium eutectoid, and the intermediate temperature, $600 \mathrm{~K}$, is a typical operating temperature for fuel cladding in a light water reactor. The initial condition for each simulation was a narrow planar hydride in the center of a zirconium domain (Fig. 3), with the overall hydrogen atomic fraction fixed at either $X_{H}^{\text {total }}=0.03$ or 0.10 ( 340 or $1230 \mathrm{wt}$ ppm). These compositions are representative of a typical hydrogen content in fuel cladding toward the 


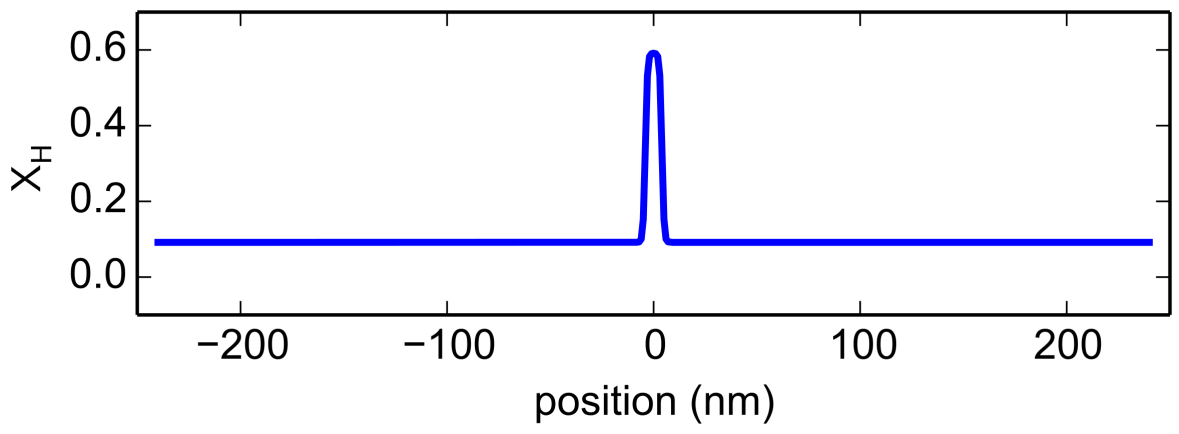

(a)

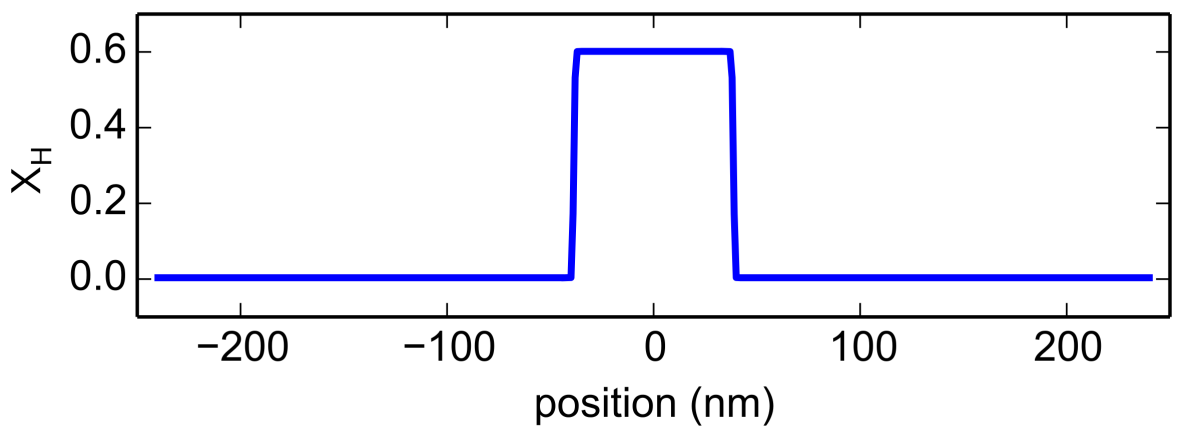

(b)

Figure 3: Line cuts of the (a) initial and (b) final hydrogen atomic fraction fields for the 2D planar hydride simulation at $550 \mathrm{~K}$ and an overall hydrogen composition of $X_{H}=0.1$. In both cases, the hydride is in the center and has a composition $X_{H}^{\delta} \approx 0.6$. In (a), the atomic fraction of hydrogen in $\alpha$-zirconium is $X=0.0917$, while in (b) it is $3.54 \times 10^{-3}$.

end of its service lifetime and a high hydrogen content that might occur during accident conditions, respectively. Two-dimensional simulations, rather than 1D, were performed for convenience (see below), with a domain size of $480 \mathrm{~nm} \times 120 \mathrm{~nm}$.

The simulations were performed with Hyrax, the phase field code developed based on the open-source finite element framework MOOSE [72--74]. Two-dimensional simulations were performed because microstructural evolution simulations will need to be performed in at least 2D, and the runtime of 2D simulations was short enough such that there was no explicit need for 1D simulations. Square, four-node quadrilateral elements were used for the mesh and linear Lagrange shape functions were used for $\eta, X$, and $\delta G / \delta X$. Adaptive 
meshing was employed to resolve the interface without incurring unnecessary computational expense, with a maximum element side length of 2.4 (in nondimensionalized units, equivalent to $2.4 \mathrm{~nm}$ ) and a minimum element side length of 0.30 , corresponding to a maximum of three levels of refinement. The BDF2 time integration scheme [75] was applied with an initial nondimensionalized time step size of $1 \times 10^{-4}$. The adaptive timestepping scheme allowed a maximum of $10 \%$ increase per time step with a maximum time step size of 0.10 . This percent increase was chosen to reduce the amount of time required to achieve equilibrium conditions; for simulations of microstructural evolution, less aggressive time adaptivity should be applied. Each multi-core simulation used a single matrix preconditioner and the Additive Schwarz Method preconditioning process, which is beneficial for parallel computing.

\section{Results and discussion}

In this section, the equilibrium phase fractions and compositions from the simulations were compared to values obtained from $G_{m i x}^{0, \alpha}$ and $G_{m i x}^{0, \delta}$ via a common tangent construction, denoted with PF and CP, respectively. An example of the initial and final conditions of the planar interface simulations is shown in Fig. 3 for $X_{H}^{\text {total }}=0.10$ at $550 \mathrm{~K}$. An initial hydride plate is present in a supersaturated zirconium matrix; at equilibrium it has grown to its final phase fraction $\left(\phi^{\delta}\right)$ and the matrix and hydride phases have achieved their final compositions ( $X_{H}^{\alpha}$ and $X_{H}^{\delta}$, respectively). The $\phi^{\delta}$ values were found for the phase field simulation by thresholding at $\eta=0.5$ and numerically integrating to find the area of the resulting spatial region.

Table 2 shows the comparisons between the simulation results and the values obtained from the common tangent construction for the phase fractions and phase compositions. In addition, Table 3 shows the difference and percent error for $\phi^{\delta}, X_{H^{\prime}}^{\delta}$ and $X_{H}^{\alpha}$ at each temperature and $X_{H}^{\text {total }}$. Overall, the results indicate that there is excellent agreement 
Table 2: Theoretical and simulated equilibrium phase characteristics of the $\alpha$-zirconium $/ \delta$-hydride system.

\begin{tabular}{|c|c|c|c|c|c|c|c|}
\hline Temperature (K) & $X_{H}^{\text {total }}$ & $X_{H}^{\alpha, C P}$ & $X_{H}^{\alpha, P F}$ & $X_{H}^{\delta, C P}$ & $X_{H}^{\delta, P F}$ & $\phi^{\delta, C P}$ & $\phi^{\delta, P F}$ \\
\hline \multirow{2}{*}{485} & 0.03 & \multirow{2}{*}{$1.07 \times 10^{-3}$} & $1.07 \times 10^{-3}$ & \multirow{2}{*}{0.605} & 0.605 & 0.0479 & 0.0483 \\
\hline & 0.10 & & $1.12 \times 10^{-3}$ & & 0.605 & 0.164 & 0.164 \\
\hline \multirow{2}{*}{550} & 0.03 & \multirow{2}{*}{$3.49 \times 10^{-3}$} & $3.49 \times 10^{-3}$ & \multirow{2}{*}{0.601} & 0.601 & 0.0443 & 0.0447 \\
\hline & 0.10 & & $3.54 \times 10^{-3}$ & & 0.601 & 0.162 & 0.163 \\
\hline \multirow{2}{*}{600} & 0.03 & \multirow{2}{*}{$7.22 \times 10^{-3}$} & $7.15 \times 10^{-3}$ & \multirow{2}{*}{0.599} & 0.598 & 0.0385 & 0.0392 \\
\hline & 0.1 & & $7.36 \times 10^{-3}$ & & 0.599 & 0.157 & 0.158 \\
\hline \multirow{2}{*}{650} & 0.03 & \multirow{2}{*}{$1.32 \times 10^{-2}$} & $1.31 \times 10^{-2}$ & \multirow{2}{*}{0.595} & 0.595 & 0.0289 & 0.030 \\
\hline & 0.10 & & $1.32 \times 10^{-2}$ & & 0.595 & 0.149 & 0.150 \\
\hline \multirow{2}{*}{750} & 0.03 & $3.00 \times 10^{-2}$ & $3.00 \times 10^{-2}$ & - & - & 0.00 & 0.00 \\
\hline & 0.10 & $3.38 \times 10^{-2}$ & $3.38 \times 10^{-2}$ & 0.587 & 0.587 & 0.120 & 0.120 \\
\hline
\end{tabular}

$X_{H}$ : overall atomic fraction of hydrogen, $X_{H}^{\alpha}$ : atomic fraction of hydrogen in $\alpha$-zirconium, $X_{H}^{\delta}$ : atomic fraction of hydrogen in $\delta$-hydride, $\phi^{\delta}$ : area fraction of $\delta$-hydride, " $\mathrm{CP}^{\prime \prime}$ : calculated from $G_{m i x}^{0, \alpha}$ and $G_{\text {mix }}^{0, \delta}$ using the common tangent construction, "PF": simulated using phase field method.

between the calculated and simulated values of $\phi^{\delta}, X_{H}^{\delta}$, and $X_{H}^{\alpha}$ over the range of $485 \mathrm{~K}$ $750 \mathrm{~K}$. The difference in $\phi^{\delta, P F}$ and $\phi^{\delta, C P}$ values for all simulations is less than or equal to 0.001; this small difference may be due to the thresholding procedure used in calculating $\phi^{\delta}$. The $X_{H}^{\delta}$ values are identical within three significant figures except for two cases, for which the error is less than $0.2 \%$. The most error occurs in $X_{H}^{\alpha}$, although it is still very low. The error in $X_{H}^{\alpha}$ is at most 5.6\% at $485 \mathrm{~K}$ and is between $1-2 \%$ at 550, 600, and $650 \mathrm{~K}$. For the $X_{H}^{\alpha, P F}$ and $X_{H}^{\alpha, C P}$ values that differ, the difference is on the order of $1 \times 10^{-4}$, and the differences generally increase slightly with increasing temperature. The error in $X_{H}^{\alpha}$ is greater than the error in $X_{H}^{\delta}$, which is due to the shape of $G_{m i x}^{0, \alpha}$ and $G_{m i x}^{0, \delta}$. Because the curvature of the energy well of $G_{m i x}^{0, \delta}$ is much greater than that of $G_{m i x}^{0, \alpha}$, small changes in $G_{m i x}^{0, \alpha}$ will have a greater effect on the equilibrium phase compositions than small changes in $G_{\text {mix }}^{0, \delta}$.

We now examine the impact of $X_{\text {bound }}$ values on the simulated $X_{H}^{\alpha}, X_{H}^{\delta}$, and $\phi^{\delta}$ values. To this end, two additional sets of simulations are performed to explore the effect of $X_{\text {bound }}$ values. The original set of simulations, detailed above, is labeled Set $\mathrm{A}$ and has 
Table 3: Difference and percent error between theoretical and simulated equilibrium phase characteristics of the $\alpha$-zirconium $/ \delta$-hydride system.

\begin{tabular}{|c|c|c|c|c|c|c|c|}
\hline Temperature (K) & $X_{H}^{\text {total }}$ & $\Delta X_{H}^{\alpha}$ & error $(\%)$ & $\Delta X_{H}^{\delta}$ & error (\%) & $\Delta \phi^{\delta}$ & error (\%) \\
\hline \hline \multirow{2}{*}{485} & 0.03 & 0.0 & 0.0 & 0.0 & 0.0 & 0.0004 & 0.8 \\
\cline { 2 - 8 } & 0.10 & $6.0 \times 10^{-5}$ & 5.6 & 0.0 & 0.0 & 0.001 & 0.6 \\
\hline \multirow{3}{*}{550} & 0.03 & 0.0 & 0.0 & 0.0 & 0.0 & 0.0004 & 0.9 \\
\cline { 2 - 8 } & 0.10 & $5.0 \times 10^{-5}$ & 1.4 & 0.001 & 0.17 & 0.001 & 0.6 \\
\hline \multirow{2}{*}{600} & 0.03 & $-7.0 \times 10^{-5}$ & 1.0 & -0.001 & 0.17 & 0.0007 & 1.8 \\
\cline { 2 - 8 } & 0.1 & $1.4 \times 10^{-4}$ & 1.8 & 0.0 & 0.0 & 0.001 & 0.6 \\
\hline \multirow{2}{*}{650} & 0.03 & $-1.0 \times 10^{-4}$ & 0.8 & 0.0 & 0.0 & 0.0011 & 3.8 \\
\cline { 2 - 8 } & 0.10 & 0.0 & 0.0 & 0.0 & 0.0 & 0.001 & 0.7 \\
\hline \multirow{2}{*}{750} & 0.03 & 0.0 & 0.0 & - & - & 0.0 & 0.0 \\
\cline { 2 - 8 } & 0.10 & 0.0 & 0.0 & 0.0 & 0.0 & 0.0 & 0.0 \\
\hline
\end{tabular}

$\Delta X_{H}^{\alpha}=X_{H}^{\alpha, P F}-X_{H}^{\alpha, C P} ; \Delta X_{H}^{\delta}=X_{H}^{\delta, P F}-X_{H}^{\delta, C P} ; \Delta \phi^{\delta}=\phi^{\delta, P F}-\phi^{\delta, C P}$.

$X_{\text {low }}^{\alpha}=0.001, X_{\text {high }}^{\alpha}=0.499, X_{\text {low }}^{\delta}=0.5$, and $X_{\text {high }}^{\delta}=0.665$. Set B improves the approximation by decreasing the lower $\alpha$ subdomain boundary composition to $X_{\text {low }}^{\alpha}=1 \times 10^{-6}$ while keeping $X_{\text {high }}^{\alpha} X_{\text {low }}^{\delta}$, and $X_{\text {high }}^{\delta}$ the same as in Set A. Finally, Set $C$ tests the sensitivity of the results on the choice of $X_{\text {bound }}$ values. The $X_{\text {bound }}$ values of Set A were changed by $5 \%$ to reduce the ranges over which $\tilde{G}_{\text {mix }}^{0, \alpha}$ and $\tilde{G}_{\text {mix }}^{0, \alpha}$ retain the CALPHAD values. Specifically, $X_{\text {low }}^{\alpha}=1.05 \times 10^{-3}, X_{\text {high }}^{\alpha}=0.474, X_{\text {low }}^{\delta}=0.525$, and $X_{\text {high }}^{\delta}=0.632$ are used.

-The percent errors in $X_{H}^{\alpha, P F}$ and $\phi^{\delta, P F}$ for the three sets with respect to $X_{H}^{\alpha, C P}$ and $X_{H}^{\delta, C P}$ are shown in Fig. 4, as well as the root-mean-square (RMS) of the error over the temperature range examined. The results for $X_{H}^{\delta, P F}$ are not shown because they show no variation within three significant figures except for Set $C, X_{H}^{\text {total }}=0.03, T=600 \mathrm{~K}$, for which $X_{H}^{\delta, P F}=0.599$. The data sets $\mathrm{A}$ and $\mathrm{B}$ are identical within three significant figures except for one value, $\phi^{\delta}$ at $600 \mathrm{~K}$ for $X_{H}^{\text {total }}=0.03$, indicating that the larger value of $X_{\text {low }}^{\alpha}=0.001$ versus $1 \times 10^{-6}$ does not affect the result. Conversely, the results of Set $\mathrm{A}$ and Set $\mathrm{C}$ differ. Most notably, the simulations at $485 \mathrm{~K}$ did not converge to equilibrium, as discussed further below. However, no obvious trends in error are evident when comparing the results of sets A and C. For some quantities, the RMS of the error is greater for Set A versus Set $C$, while for others, it is less; the maximum RMS error is less than $3 \%$. 
The two simulations for Set $\mathrm{C}$ at $485 \mathrm{~K}$ failed to reach equilibrium. The $\phi^{\delta}$ values for both simulations attained $99 \%$ of $\phi^{\delta, C P}$, but the solver was unable to reduce the residual further. Because the simulations at higher temperatures did not experience this issue, it appears that the choice of $X_{\text {low }}^{\alpha}$ is the cause. While $X_{\text {low }}^{\alpha}=1.05 \times 10^{-3}$ is less than $X_{H}^{\alpha, C P}=1.07 \times 10^{-3}$, the function approximation may occur at a value too close to $X_{H}^{\alpha, C P}$. The curvature of the approximated function $G_{\text {low }}^{\alpha, \text { Taylor }}$ is less than the original function $G_{m i x}^{0, \alpha}$ which may interfere with the ability of the numerical solver to determine the solution near equilibrium. To avoid this issue, we suggest that $X_{\text {low }}^{\alpha}$ be chosen around $90 \%$ of $X_{H}^{\alpha, C P}$. A similar consideration is likely necessary for $X_{\text {high }}^{\delta}$. In general, we recommend conducting planar interface simulations to quantify the error in a given system when applying the proposed approximation method.

The proposed approximation method is advantageous over other techniques of incorporating CALPHAD-based free energy expressions into phase field simulations because it is both simple to implement and addresses the issues in using these free energies. One alternative method, linking the phase field software directly to a thermodynamic database $[26,50,51]$, requires an interface to database software, potentially costly software licensing, and additional computational expense to query the database at each mesh point and each time step. Another alternative method, polynomial approximation of the free energy expressions [35, 52--55, 76], requires more effort than the proposed method. For example, one must make choices among many options, e.g., various fitting and error weighting schemes, when performing a polynomial fit. In contrast, the proposed approximation method allows the free energy expression to be directly incorporated into the phase field software with small error and little effort. While the implementation was performed for a finite element framework, the method may be used with other numerical methods such as the finite difference method.

Figure 5 illustrates how the approximation method handles the extreme case of a negative atomic fraction value. Two $\delta$-hydride precipitates were seeded into an $\alpha$-zirco- 


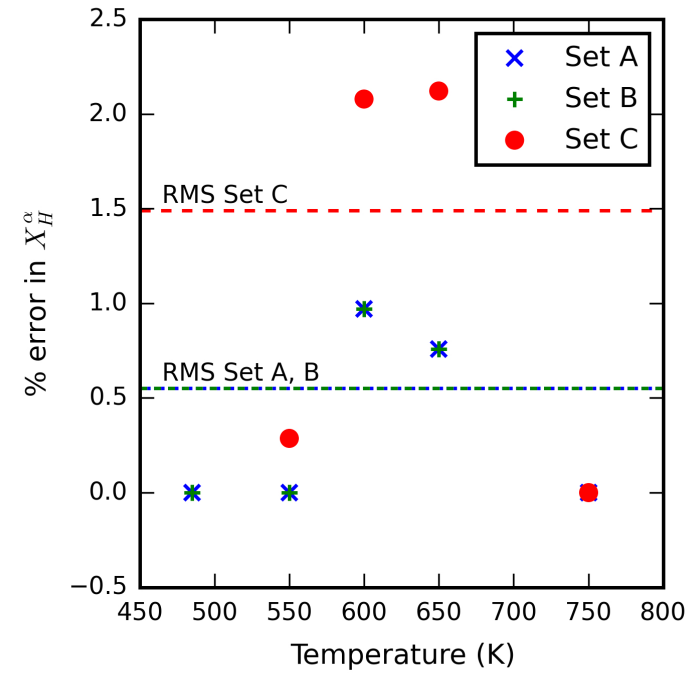

(a)

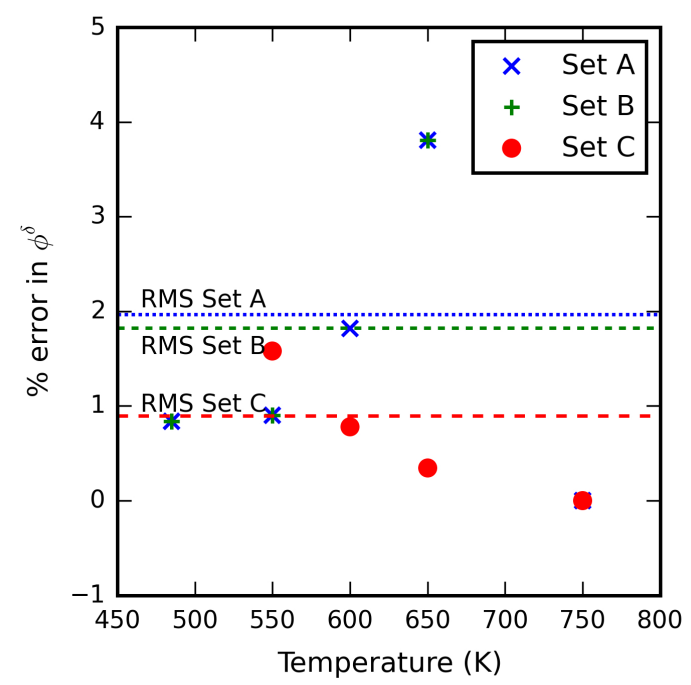

(c)

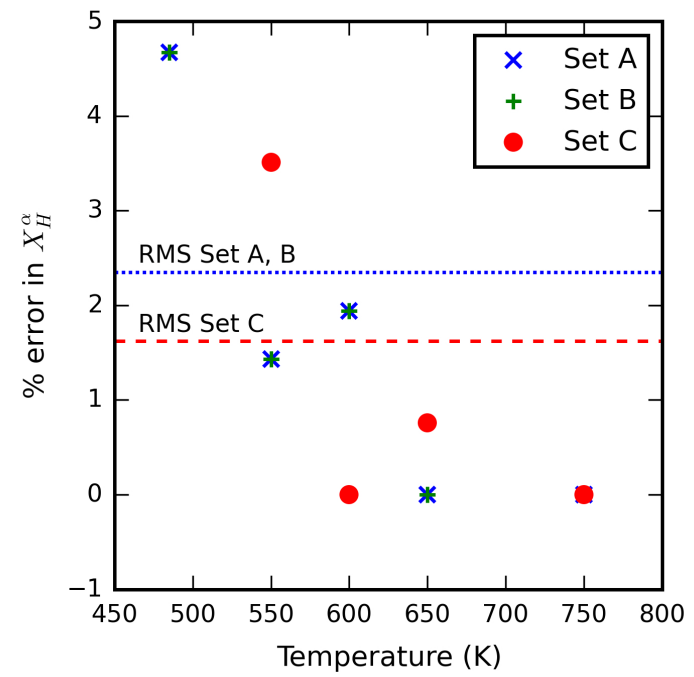

(b)

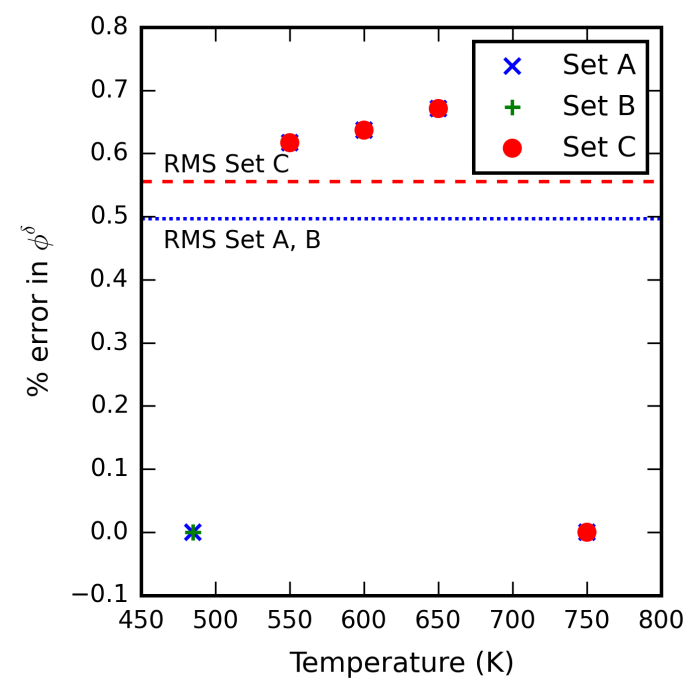

(d)

Figure 4: The percent error in $X_{H}^{\alpha, P F}$ and $\phi^{\delta, P F}$ versus $X_{H}^{\alpha, C P}$ and $\phi^{\delta, C P}$ for three sets of simulations with different $X_{\text {bound }}$ values. The root-mean-square value of the error over the temperature range is indicated by the dashed lines. The parameter values for each set are given in the text. Subfigures (a) and (c) illustrate the percent error in $X_{H}^{\alpha}$ and $\phi^{\delta}$ for $X_{H}^{\text {total }}=0.03$, respectively. Subfigures (b) and (d) illustrate the percent error in $X_{H}^{\alpha}$ and $\phi^{\delta}$ for $X_{H}^{\text {total }}=0.10$, respectively. 


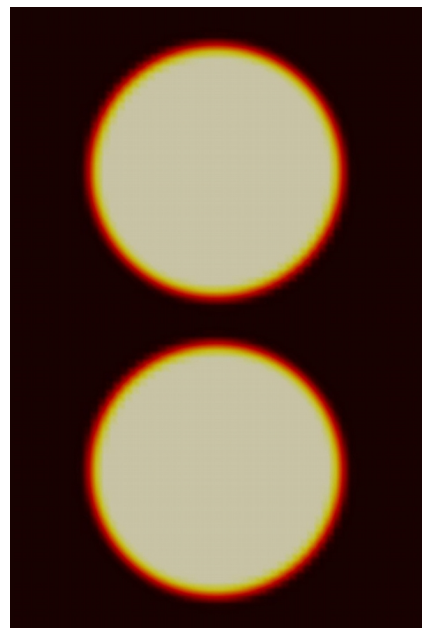

(a)

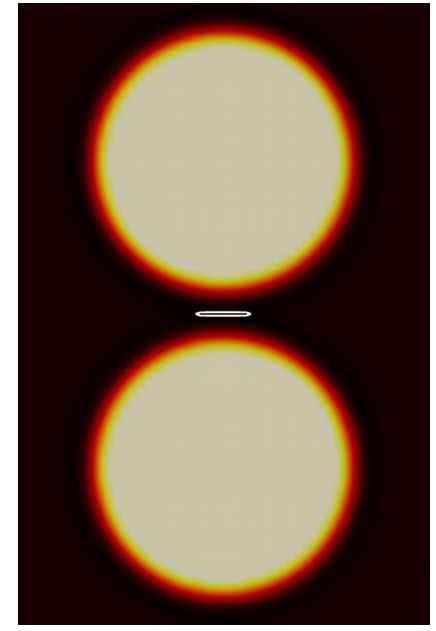

(b)

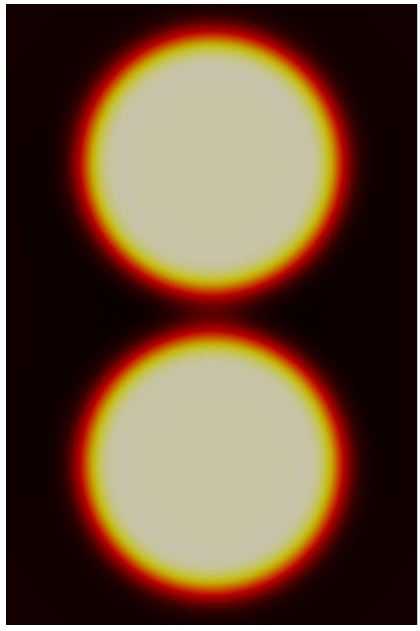

$\mathrm{X}_{\mathrm{H}}$

$-0.62$

(c)

Figure 5: An example of precipitate growth and hydrogen depletion. (a) The initial condition, in which two $\delta$-hydride precipitates were seeded into an $\alpha$-zirconium matrix with a matrix composition of $X=0.015$ at $485 \mathrm{~K}$. (b) After two time steps, depletion regions have started to form around both precipitates. The white contour line indicates a small region between the precipitates in which the hydrogen atomic fraction has become slightly negative. (c) After thirty time steps, longer-range diffusion has supplied additional solute, and the hydrogen atomic fraction has increased such that the solute value is positive everywhere.

nium matrix with a matrix composition of $X=0.015$ at $485 \mathrm{~K}$, and $X_{\text {low }}^{\alpha}=1 \times 10^{-6}$ (Fig.

5a). Both hydride precipitates induce a depletion zone around themselves as they grow.

During the first several time steps, in which the system rapidly adjusts from the initial conditions, the atomic fraction of hydrogen between the two hydrides becomes slightly negative (Fig. 5b). This transient is resolved when longer-range diffusion supplies additional solute, and physical behavior is quickly reestablished (Fig. 5c). The approximation method results in a free energy that is well behaved even when the evolution equations of the phase field model temporarily drive the system toward unphysical values. In this scenario, it is acceptable to allow small, transient values of negative concentration because the driving force and kinetics of the system tends to correct them. On the other hand, if we were to simply use the CALPHAD free energy, the code will yield undefined values. 


\section{Conclusions}

The proposed free energy approximation method is a simple and efficient technique to overcome the numerical challenges encountered during phase field simulations that incorporate CALPHAD-based free energies of mixing. The free energies are transformed into piecewise functions composed of three subfunctions that are defined on subdomains of solute composition. Low and high subdomain boundary compositions are chosen within the composition domain over which each free energy is defined. The subdomain boundary compositions are chosen to be sufficiently outside the composition range expected to be encountered in the simulation. These values may be chosen to avoid unwanted behavior in the free energy or its derivatives, such as an undefined energy at a composition that will be evaluated within the phase field model, or a free energy curvature that will yield a negative mobility. At each boundary composition, the free energy is approximated as a second-order Taylor expansion of the free energy around the boundary composition. The free energy between the low and high subdomain boundary compositions retains the original form, while outside this region, the Taylor expansions are substituted. The resulting free energy is second-order differentiable over the entire range of structural order parameter and composition values encountered within the simulation.

A phase field model for the $\alpha$-zirconium $/ \delta$-hydride system was employed to demonstrate the free energy approximation method. Planar interface simulations were performed to verify the approximation method over a range of temperatures. The compositions of the $\alpha$ and $\delta$ phases as well as the $\delta$ phase fraction obtained from the phase field simulations were compared to the values calculated from the common tangent construction of the original CALPHAD free energies. The results indicate that the approximation method does not appreciably affect the equilibrium compositions and phase fractions of the $\alpha$-zirconium $/ \delta$ hydride system. In addition, the error in the compositions of the $\alpha$ and $\delta$ phases as well as the $\delta$ phase fraction obtained from the phase field simulations were examined. We found that for the $\alpha$-zirconium $/ \delta$-hydride system, $X_{\text {low }}^{\alpha}$ should be approximately $10 \%$ less than 
equilibrium composition of the $\alpha$ phase to ensure that simulations can run to equilibrium.

We also found that while the simulation results do change slightly with a $5 \%$ variation in $X_{\text {bound }}$ values, the RMS of the error is less than 3\% for all quantities examined as long as the aforementioned condition on $X_{\text {low }}^{\alpha}$ is met.

The approximation method presented here is general and can be straightforwardly applied to other materials systems. It is particularly useful for CALPHAD free energies developed using sublattice models, because these energies may not be defined over the entire range of compositions that would be encountered in the phase field model. As the free energies of many substitutional and interstitial solutions are modeled using sublattice models, this method will increase the applicability of phase field models to a wide range of alloy systems. In addition, the method reduces the stiffness of the equations at the composition domain boundaries, which may improve numerical behavior. However, in the WBM formulation, the diffuse interface width that results from using physically appropriate interface energies can be constrained to several nanometers due to the presence of an inherent energy barrier in the free energy. Thin interface widths increase the computational cost, possibly rendering large-scale simulations prohibitively expensive. Thus, the KKS phase field model formulation may be better suited for incorporating CALPHAD-based free energies so that the interfacial thickness may be readily adjusted.

\section{Acknowledgements}

This research was supported by the Consortium for Advanced Simulation of Light Water Reactors (www.casl.gov), an Energy Innovation Hub (http:/ /www.energy.gov/hubs) for Modeling and Simulation of Nuclear Reactors under U.S. Department of Energy Contract No. DE-AC05-00OR22725. The simulations were performed using the high performance computation resources Fission and Quark at Idaho National Laboratory (INL). Many thanks to the MOOSE team at INL for the continuing, generous help and support with MOOSE. In addition, many thanks to Michael Tonks at INL for his help and experience with phase field modeling using MOOSE. Finally, we gratefully acknowledge the discussions with Dr. Olle Heinonen at Argonne National Laboratory. 


\section{Appendix}

This appendix provides the parameterizations for the free energies of mixing appearing in Eqs. 14 and 15. The parameterizations for $G_{\mathrm{ZrH}}^{0, h c p}, G_{\mathrm{ZrH}_{2}}^{0, f f c} L_{f c c^{\prime}}^{0}$ and $L_{f c c}^{1}$ are supplied by Ref. [56], and the parameterizations for $G_{Z r}^{0, h c p}$ and $G_{Z r}^{0, f c c}$ are from Ref. [69]. We parameterized $G_{\mathrm{H}_{2}}^{0, \text { gas }}$, which is needed to calculate $G_{\mathrm{ZrH}}^{0, h c p}$ and $G_{\mathrm{ZrH}_{2}}^{0, f f c}$, using the data from Ref. [70].

$$
\begin{gathered}
G_{Z r}^{0, h c p}(T)=-7827.595+125.64905 T-24.1618 T \ln (T)-4.37791 \times 10^{-3} T^{2}+34971 T^{-1} \mathrm{~J} / \mathrm{mol} \\
G_{Z r}^{0, f c c}(T)=-227.595+124.74905 T-24.1618 T \ln (T)-4.37791 \times 10^{-3} T^{2}+34971 T^{-1} \mathrm{~J} / \mathrm{mol} \\
G_{H_{2}}^{0, g a s}(T)=8055.34-243.79 T+18.313 T \ln (T)-0.034513 T^{2}-734182 T^{-1} \mathrm{~J} / \mathrm{mol} \\
G_{Z r H}^{0, h c p}(T)=-45965+41.6 T+G_{Z r}^{0, h c p}(T)+\frac{1}{2} G_{H_{2}}^{0, g a s}(T) \\
G_{Z r H_{2}}^{0, f c c}(T)=-170490+208.2 T-9.47 T \ln (T)+G_{Z r}^{0, h c p}(T)+G_{H_{2}}^{0, g a s}(T) \\
L_{f c c}^{0}=14385-6.0 T \\
L_{f c c}^{1}=-106445+87.3 T
\end{gathered}
$$

[1] Yongwoo Kwon, Katsuyo Thornton, and Peter W Voorhees. Coarsening of bicontinuous structures via nonconserved and conserved dynamics. Physical Review E, 75(2): 021120, 2007.

[2] Y Kwon, K Thornton, and PW Voorhees. The topology and morphology of bicontinuous interfaces during coarsening. EPL (Europhysics Letters), 86(4):46005, 2009.

[3] DJ Seol, SY Hu, YL Li, J Shen, KH Oh, and LQ Chen. Three-dimensional phasefield modeling of spinodal decomposition in constrained films. Metals and Materials International, 9(1):61--66, 2003.

[4] John W Cahn. On spinodal decomposition. Acta Metallurgica, 9(9):795--801, 1961. 
[5] H-J Diepers, C Beckermann, and I Steinbach. Simulation of convection and ripening in a binary alloy mush using the phase-field method. Acta Materialia, 47(13):3663--3678, 1999.

[6] R Mendoza, I Savin, K Thornton, and PW Voorhees. Topological complexity and the dynamics of coarsening. Nature Materials, 3(6):385--388, 2004.

[7] WJ Boettinger, JA Warren, C Beckermann, and A Karma. Phase-field simulation of solidification 1. Annual Review of Materials Research, 32(1):163--194, 2002.

[8] James A Warren and William J Boettinger. Prediction of dendritic growth and microsegregation patterns in a binary alloy using the phase-field method. Acta Metallurgica et Materialia, 43(2):689--703, 1995.

[9] James A Warren, Ryo Kobayashi, Alexander E Lobkovsky, and W Craig Carter. Extending phase field models of solidification to polycrystalline materials. Acta Materialia, 51(20):6035--6058, 2003.

[10] SM Wise, JS Lowengrub, JS Kim, K Thornton, PW Voorhees, and WC Johnson. Quantum dot formation on a strain-patterned epitaxial thin film. Applied Physics Letters, 87(13):133102, 2005.

[11] Yu U Wang, Yongmei M Jin, and Armen G Khachaturyan. Phase field microelasticity modeling of surface instability of heteroepitaxial thin films. Acta Materialia, 52(1): 81--92, 2004.

[12] Long-Qing Chen. Phase-field method of phase transitions/domain structures in ferroelectric thin films: A review. Journal of the American Ceramic Society, 91(6):1835-$1844,2008$.

[13] DJ Seol, SY Hu, ZK Liu, LQ Chen, SG Kim, and KH Oh. Phase-field modeling of stress-induced surface instabilities in heteroepitaxial thin films. Journal of Applied Physics, 98(4):044910, 2005.

[14] Nele Moelans, Bart Blanpain, and Patrick Wollants. An introduction to phase-field modeling of microstructure evolution. Calphad, 32(2):268--294, 2008.

[15] Ingo Steinbach. Phase-field models in materials science. Modelling and Simulation in Materials Science and Engineering, 17(7):073001, 2009.

[16] Britta Nestler and Abhik Choudhury. Phase-field modeling of multi-component systems. Current Opinion in Solid State and Materials Science, 15(3):93--105, 2011.

[17] C. Shen and Y. Wang. Fundamentals of Modeling for Metals Processing, volume 22A of ASM Handbook. ASM International, 2009.

[18] Long-Qing Chen. Phase-field models for microstructure evolution. Annual Review of Materials Research, 32(1):113--140, 2002. 
[19] Samuel M Allen and John W Cahn. A microscopic theory for antiphase boundary motion and its application to antiphase domain coarsening. Acta Metallurgica, 27(6): 1085--1095, 1979.

[20] Hans Leo Lukas, Suzana G Fries, and Bo Sundman. Computational Thermodynamics: the Calphad Method, volume 131. Cambridge University Press Cambridge, 2007.

[21] Nigel Saunders and A Peter Miodownik. CALPHAD (Calculation of Phase Diagrams): A Comprehensive Guide, volume 1. Elsevier, 1998.

[22] P-R Cha, D-H Yeon, and J-K Yoon. A phase field model for isothermal solidification of multicomponent alloys. Acta Materialia, 49(16):3295--3307, 2001.

[23] Dong-Hee Yeon, Pil-Ryung Cha, and Jong-Kyu Yoon. A phase field study for ferriteaustenite transitions under para-equilibrium. Scripta Materialia, 45(6):661--668, 2001.

[24] Irina Loginova, John Agren, and Gustav Amberg. On the formation of widmanstatten ferrite in binary Fe-C - Phase-field approach. Acta Materialia, 52(13):4055--4063, 2004.

[25] J Rudnizki, B Bottger, U Prahl, and W Bleck. Phase-field modeling of austenite formation from a ferrite plus pearlite microstructure during annealing of cold-rolled dual-phase steel. Metallurgical and Materials Transactions A, 42(8):2516--2525, 2011.

[26] U Grafe, B Bottger, J Tiaden, and SG Fries. Coupling of multicomponent thermodynamic databases to a phase field model: application to solidification and solid state transformations of superalloys. Scripta Materialia, 42(12):1179--1186, 2000.

[27] JZ Zhu, ZK Liu, V Vaithyanathan, and LQ Chen. Linking phase-field model to CALPHAD: Application to precipitate shape evolution in Ni-base alloys. Scripta Materialia, 46(5):401--406, 2002.

[28] JZ Zhu, T Wang, AJ Ardell, SH Zhou, ZK Liu, and LQ Chen. Three-dimensional phase-field simulations of coarsening kinetics of $\gamma^{\prime}$ particles in binary $\mathrm{Ni}-\mathrm{Al}$ alloys. Acta Materialia, 52(9):2837--2845, 2004.

[29] Kaisheng $\mathrm{Wu}, \mathrm{YA}$ Chang, and Yunzhi Wang. Simulating interdiffusion microstructures in Ni-Al-Cr diffusion couples: A phase field approach coupled with CALPHAD database. Scripta Materialia, 50(8):1145--1150, 2004.

[30] JC Wang, M Osawa, T Yokokawa, H Harada, and M Enomoto. Modeling the microstructural evolution of Ni-base superalloys by phase field method combined with CALPHAD and CVM. Computational Materials Science, 39(4):871--879, 2007.

[31] Tomonori Kitashima, Jincheng Wang, and Hiroshi Harada. Phase-field simulation with the CALPHAD method for the microstructure evolution of multi-component Ni-base superalloys. Intermetallics, 16(2):239--245, 2008.

[32] N Warnken, D Ma, A Drevermann, Roger C Reed, SG Fries, and I Steinbach. Phasefield modelling of as-cast microstructure evolution in nickel-based superalloys. Acta Materialia, 57(19):5862--5875, 2009. 
[33] YH Wen, JV Lill, SL Chen, and JP Simmons. A ternary phase-field model incorporating commercial CALPHAD software and its application to precipitation in superalloys. Acta Materialia, 58(3):875--885, 2010.

[34] B Bottger, J Eiken, and I Steinbach. Phase field simulation of equiaxed solidification in technical alloys. Acta Materialia, 54(10):2697--2704, 2006.

[35] SY Hu, J Murray, H Weiland, ZK Liu, and LQ Chen. Thermodynamic description and growth kinetics of stoichiometric precipitates in the phase-field approach. Calphad, 31 (2):303--312, 2007.

[36] Hiroki Kobayashi, Machiko Ode, Seong Gyoon Kim, Won Tae Kim, and Toshio Suzuki. Phase-field model for solidification of ternary alloys coupled with thermodynamic database. Scripta Materialia, 48(6):689--694, 2003.

[37] RS Qin and ER Wallach. A phase-field model coupled with a thermodynamic database. Acta Materialia, 51(20):6199--6210, 2003.

[38] RS Qin, ER Wallach, and RC Thomson. A phase-field model for the solidification of multicomponent and multiphase alloys. Journal of Crystal Growth, 279(1):163--169, 2005.

[39] I Steinbach, B Bottger, J Eiken, N Warnken, and SG Fries. Calphad and phase-field modeling: A successful liaison. Journal of Phase Equilibria and Diffusion, 28(1):101--106, 2007.

[40] Y Mishin, WJ Boettinger, JA Warren, and GB McFadden. Thermodynamics of grain boundary premelting in alloys. I. Phase-field modeling. Acta Materialia, 57(13):3771-$3785,2009$.

[41] Jeroen Heulens, Bart Blanpain, and Nele Moelans. Phase field modeling of the crystallization of $\mathrm{FeOx}-\mathrm{SiO} 2$ melts in contact with an oxygen-containing atmosphere. Chemical Geology, 290(3):156--162, 2011.

[42] J-L Fattebert, ME Wickett, and PEA Turchi. Phase-field modeling of coring during solidification of Au-Ni alloy using quaternions and CALPHAD input. Acta Materialia, 62:89--104, 2014.

[43] Qing Chen, Ning Ma, Kaisheng Wu, and Yunzhi Wang. Quantitative phase field modeling of diffusion-controlled precipitate growth and dissolution in Ti-Al-V. Scripta Materialia, 50(4):471--476, 2004.

[44] HM Singer, Irina Singer, and A Jacot. Phase-field simulations of $\alpha$ to $\gamma$ precipitations and transition to massive transformation in the Ti-Al alloy. Acta Materialia, 57(1): 116--124, 2009.

[45] MS Park, SL Gibbons, and R Arroyave. Phase-field simulations of intermetallic compound evolution in $\mathrm{Cu} / \mathrm{Sn}$ solder joints under electromigration. Acta Materialia, 61(19):7142--7154, 2013. 
[46] K. Grönhagen, J. Ågren, and M. Odén. Phase-field modelling of spinodal decomposition in tialn including the effect of metal vacancies. Scripta Materialia, 95:42 -- 45, 2015.

[47] A. A. Wheeler, W. J. Boettinger, and G. B. McFadden. Phase-field model for isothermal phase transitions in binary alloys. Physical Review A, 45(10):7424--7439, 1992.

[48] S. G. Kim, W. T. Kim, and T. Suzuki. Phase-field model for binary alloys. Physical Review E, 60(6):7186--7197, 1999.

[49] Michael J. Welland, Dieter Wolf, and Jonathan E. Guyer. Multicomponent phase-field model for extremely large partition coefficients. Phys. Rev. E, 89:012409, Jan 2014. doi: 10.1103/PhysRevE.89.012409.

[50] Hua Xiong, Zhiheng Huang, Zhiyong $\mathrm{Wu}$, and Paul P Conway. A generalized computational interface for combined thermodynamic and kinetic modeling. Calphad, 35(3):391--395, 2011.

[51] Lijun Zhang, Matthias Stratmann, Yong Du, Bo Sundman, and Ingo Steinbach. Incorporating the CALPHAD sublattice approach of ordering into the phase-field model with finite interface dissipation. Acta Materialia, 88:156--169, 2015.

[52] Akinori Yamanaka, Tomohiro Takaki, and Yoshihiro Tomita. Simulation of austeniteto-ferrite transformation in deformed austenite by crystal plasticity finite element method and multi-phase-field method. ISIJ international, 52(4):659--668, 2012.

[53] Kumar Ankit, Abhik Choudhury, Cheng Qin, Sebastian Schulz, Malte McDaniel, and Britta Nestler. Theoretical and numerical study of lamellar eutectoid growth influenced by volume diffusion. Acta Materialia, 61(11):4245--4253, 2013.

[54] T Philippe, D Erdeniz, DC Dunand, and PW Voorhees. A phase-field study of the aluminizing of nickel. Philosophical Magazine, (ahead-of-print):1--13, 2015.

[55] Hong Liu, Yipeng Gao, JZ Liu, YM Zhu, Yunzhi Wang, and JF Nie. A simulation study of the shape of $\beta$ precipitates in Mg-Y and Mg-Gd alloys. Acta Materialia, 61(2): 453--466, 2013.

[56] N Dupin, I Ansara, C Servant, C Toffolon, C Lemaignan, and JC Brachet. A thermodynamic database for zirconium alloys. Journal of Nuclear Materials, 275(3):287--295, 1999.

[57] C. M. Elliott, D. A. French, and F. A. Milner. A second order splitting method for the cahn-hilliard equation. Numerische Mathematik, 54:575--590, 1989.

[58] L. Zhang, M. R. Tonks, D. Gaston, J. W. Peterson, D. Andrs, P. C. Millett, and B. S. Biner. A quantitative comparison between $\mathrm{C} 0$ and $\mathrm{C} 1$ elements for solving the CahnHilliard equation. Journal of Computational Physics, 236:74--80, 2013. 
[59] ATW Barrow, A Korinek, and MR Daymond. Evaluating zirconium--zirconium hydride interfacial strains by nano-beam electron diffraction. Journal of Nuclear Materials, 432(1):366--370, 2013.

[60] M. P. Puls. Hydrogen-induced delayed hydride cracking: 1. Strain energy effects on hydrogen solubility. Technical Report AECL-6302, Atomic Energy of Canada Limited, 1978.

[61] Ludovic Thuinet and Remy Besson. Ab initio study of competitive hydride formation in zirconium alloys. Intermetallics, 20:24--32, 2012.

[62] David A Porter and Kenneth E Easterling. Phase Transformations in Metals and Alloys, (Revised Reprint). CRC Press, 1992.

[63] GJC Carpenter. The dilatational misfit of zirconium hydrides precipitated in zirconium. Journal of Nuclear Materials, 48(3):264--266, 1973.

[64] R.W. Balluffi, S. Allen, and W.C. Carter. Kinetics of Materials. Wiley-Interscience, Hoboken, New Jersey, 2005. ISBN 9780471749301.

[65] J. J. Kearns. Diffusion coefficient of hydrogen in $\alpha$ zirconium, Zircaloy-2 and Zircaloy4. Journal of Nuclear Materials, 43:330--338, 1972.

[66] G Majer, W Renz, and RG Barnes. The mechanism of hydrogen diffusion in zirconium dihydrides. Journal of Physics: Condensed Matter, 6(15):2935, 1994.

[67] M. Hillert and L.-I. Staffansson. The regular solution model for stoichiometric phases and ionic melts. Acta Chemica Scandinavica, 24:3618--3626, 1970.

[68] B. Sundman and J. Agren. A regular solution model for phases with several components and sublattices, suitable for computer applications. Journal of Physics and Chemistry of Solids, 42:297--301, 1981.

[69] A. T. Dinsdale. SGTE data for pure elements. CALPHAD, 15:317--425, 1991.

[70] J. D. Cox, D. D. Wagman, and V. A. Medvedev. CODATA: Key Values for Thermodynamics. Hemisphere Publishing Corporation, New York, 1989.

[71] V. Vaithyanathan, C. Wolverton, and L. Q. Chen. Multiscale modeling of $\theta^{\prime}$ precipitation in Al-Cu binary alloys. Acta Materialia, 52:2973--2987, 2004.

[72] Derek R Gaston, John W Peterson, Cody J Permann, David Andrs, Andrew E Slaughter, and Jason M Miller. Continuous integration for concurrent computational framework and application development. Journal of Open Research Software, 2(1):e10, 2014.

[73] Derek R Gaston, Cody J Permann, John W Peterson, Andrew E Slaughter, avid Andrš, Yaqi Wang, Michael P Short, Danielle M Perez, Michael R Tonks, Javier Ortensi, et al. Physics-based multiscale coupling for full core nuclear reactor simulation. Annals of Nuclear Energy, 2014. 
[74] Michael R. Tonks, Derek Gaston, Paul C. Millett, David Andrs, and Paul Talbot. An object-oriented finite element framework for multiphysics phase field simulations. Computational Materials Science, 51(1):20--29, JAN 2012. doi: 10.1016/j.commatsci.2011.07.028.

[75] A. Iserles. A First Course in the Numerical Analysis of Differential Equations. Cambridge University Press, Cambridge, UK, 1996.

[76] Amer Malik, Gustav Amberg, Annika Borgenstam, and John Ågren. Effect of external loading on the martensitic transformation--a phase field study. Acta Materialia, 61(20): 7868--7880, 2013. 